\title{
Perianal Region
}

National Cancer Institute

\section{Source}

National Cancer Institute. Perianal Region. NCI Thesaurus. Code C99148.

The skin area around the anus. 